\title{
LERNEN UND KOMMUNIKATIVES HANDELN IM ZEITALTER DES INTERNET
}

\author{
Werner Westphal \\ Greifswald / Szczecin
}

Received: 28 March, 2003

\begin{abstract}
The present paper focuses on different aspects of e-learning. Differences between
traditional learning processes on the basis of written texts and e-learning on the basis of hypertext are discussed in this context. The main differences are to be found in the way of transportation and reception and the kind of presentation of information (multi-medial). This, of course, has a lot of consequences for both people's socialization and the teaching process itself. Young people in particular need help to find effective ways of using the new possibilities of information transfer. Support should be provided by specially qualified tutors. One of the important conclusions is that e-learning is a new challenge for studies in different fields as well as a challenge and chance for interdisciplinary cooperation.
\end{abstract}

Keywords: e-learning, multimedia, socialization, interdisciplinary cooperation

Meine sehr verehrten Damen und Herren, ich bedanke mich im Namen meiner Organisation, des Europäischen Schulverbandes (FEDE) herzlich für diese Einladung. Als NGO mit Konsultativstatus beim Europarat nehmen wir unsere Verantwortung für das Europa von morgen sehr ernst. „To build a Europe of knowledge", mit diesem Slogan beschreibt das Generaldirektorat für Bildung und Kultur seinen Auftrag.

Es ist dies eine treffende Beschreibung dafür, wie ein wettbewerbfähiges Europa gestaltet sein soll. Wissen wird dafür der entscheidende Rohstoff sein. Die Vorteile dieses „Rohstoffes“ liegen auf der Hand, er ist regenerierbar und lässt sich leicht ,transportieren“. Allerdings müssen sowohl die Transportwege als auch die Transportbedingungen stimmen. Die neuen Informations- und Kommunikationstechnologien, kurz IKT bieten uns vorher nicht gekannte Möglichkeiten des Informationstransfers und völlige neue Möglichkeiten des effektiven Wissenserwerbs. Damit sind wir beim Themen dieser Konferenz.

Die Europäische Kommission hat am 21.2.2002 einen
Zwischenbericht über die Rolle des E- Learning in Europa vorgelegt (vgl. Zwischenbericht gemäß der Entschließung des Rates vom 13. Juli 2001, SEK 2001: 236).

Unter dem Titel: „E- Learning: Gedanken zur Bildung von Morgen" wird die derzeitige Situation einer Analyse unterzogen. Ausgangspunkt ist das auf der Ratssitzung im März 2000 formulierte ehrgeizige Ziel: Europa innerhalb von 10 Jahren zum wettbewerbsfähigsten und dynamischsten wissensbasierten Wirtschaftsraum der Welt zu machen.

Diese Zielsetzung spricht eindeutig dafür, dass die Kommission erkannt hat, welche Bedeutung der Einsatz von IKT im Bildungsbereich für die wirtschaftliche Konkurrenzfähigkeit des alten Kontinents hat.

In diesem Zusammenhang gewinnen die Ergebnisse der PISA-Studie an besonderer Bedeutung. Wenn es also stimmt, dass das Wohl und Wehe ganzer Volkswirtschaften davon abhängt, wie es gelingt, den Rohstoff Wissen als Produktivfaktor einzusetzen, dann - so muss man leider feststellen - haben die Deutschen 
auf diesem entscheidenden Feld des Wettbewerbs große Versäumnisse zugelassen.

Nach der jüngsten OECD-Studie liegen die Ausgaben für Bildung in Deutschland immer noch unter dem OECD-Durchschnitt $(5,6 \%$ zu 5,8 \% BiP, OECDDurchschnitt, Quelle „Ostseezeitung“, 30.10.2002). Wenngleich davon auszugehen ist, dass nunmehr auch in Deutschland $100 \%$ der Schulen am Netz sind, so ist damit noch nichts über die Qualität der Nutzung der neuen Medien gesagt.

Zurecht wird im Zwischenbericht konstatiert : „Der erfolgreiche Einsatz der neuen Inhalte und Dienstleistungen hängt weitgehend von der Qualität des Unterrichts und dem Engagement der Lehrer ab" (a.a.O., 5).

Die Einsatzmöglichkeiten und methodische Gestaltung des E- Learning sind erst in Ansätzen wissenschaftlich ausgelotet. Dennoch, das virtuelle Klassenzimmer ist schon heute Realität. Es ermöglicht flexible Unterrichtszeiten und schnellen Informationstransfer. Benachteiligte und ältere Menschen können nun den Traum vom lebenslangen Lernen Realität werden lassen. In England hat man „Learning Centers“ and „Learning Partnerchips“ gebildet, um möglichst vielen Menschen die Teilhabe am gesellschaftlichen Wissenstransfer zu ermöglichen. Netzwerke mit Partnern in aller Welt multiplizieren die positiven Effekte.

Hier erweist sich die Nutzung der neuen Medien auch als völkerverbindendes Regulativ oder als Mittel der „Kohäsion“, um in der Terminologie der Europäischen Kommission zu bleiben.

Nach einer Eurobarometer - Erhebung vom Frühjahr 2002 benutzen 9 von 10 Lehrkräften in Europa einen Computer für die Vorbereitung und Durchführung des Unterrichts. 7 von 10 verfügten zu Hause über einen Internetanschluss. Insgesamt wird eingeschätzt, dass es einen Mangel an digitalen Inhalten gibt.

Im E-Learning Aktionsplan der Europäischen Kommission werden folgende Sachbereiche besonders betont: Lebende Sprachen, Wissenschaft, Technologie, Gesellschaft, Kunst u. Kultur, aktive Staatsbürgerschaft (a.a.O.: 11).

Ebenso gibt es einen steigenden Bedarf für methodische Anleitungen. Im Zentrum steht dabei die komplementäre Verbindung von traditionellen Lehrmethoden mit dem E- Learning. Bei Lehrern und Lernern geht es um die Entwicklung und Förderung einer spezifischen digitalen Kompetenz. Dies wird ausdrücklich als tragender Teil einer Europäischen Beschäftigungsstrategie angesehen. Geplant ist auch ein europäisches Diplom für IT- Fertigkeiten.

Bei allen diesen Fakten darf nicht der gravierende Einfluss der neuen Medien auf das kommunikative Verhalten der Menschen übersehen werden. Damit verbunden sind praktische Fragen der Methodik der Wissensvermittlung und Kompetenzentwicklung. ELearning- Prozesse werden ebenso gravierenden Einfluss auf die Sozialisation und das Handeln der Menschen haben. Die nachfolgenden Überlegungen sollen einen Beitrag zur Diskussion dieser Fragen liefern

\section{Lernen im Spannungsfeld von Realität und Virtualität}

Der Begriff des Lernens ist terminologisch schwer fassbar. Die Psychologen bringen Lernen und Lernprozesse in der Regel mit Verhalten und Verhaltensänderung in Verbindung. Und tatsächlich ist Lernen für die Justierung menschlichen Verhaltens und Handelns eine unabdingbare Voraussetzung. Wir betonen im Zusammenhang mit unserem Thema bewusst den pädagogischen und informationstheoretischen Aspekt des Lernens und folgen Klix, der feststellt: "Lernen besteht in der Ausbildung oder Korrektur von individuellem Gedächtnisbesitz" [1].

Wesentliche Voraussetzungen dafür sieht er : in umgebungsgemäßen Wahrnehmungsleistungen, den umgebungsangepassten Steuerungen motorischer Verhaltenseinheiten,

in der Anregung von Gedächtnisbesitz und inneren Bewertungen..." (a.a.O.: 350).

Dies trifft prinzipiell auch auf E-Learning-Prozesse zu.

Wir definieren Lernen als (selbst- oder fremdgesteuerten) Prozess der kognitiven Verarbeitung von Informationen zu Wissen im Sinne des Erwerbs von (zumindest angestrebten) langfristigem Gedächtnisbesitz (vgl. dazu die Literaturempfehlung bei [2]).

Informationen werden zu Wissen, indem sie in ein vorhandenes Speichersystem integriert werden und eine Zweckorientierung erhalten. Dies setzt die selektive Verarbeitung von Informationen voraus. Dabei soll das Wissen möglichst dauerhaft und in einer Weise gespeichert werden, dass es schnell reproduzierbar ist (Speicherung im Langzeitgedächtnis) (vgl. dazu [3]). Lernprozesse bilden die Basis für Verhaltensänderungen und zielgerichtetes Handeln. So sind z. B. Erfolge bei der Erforschung von Naturgesetzen an bestimmte Wissensvoraussetzungen gebunden.

Praktischer Zweck von Lernprozessen im Sinne von Wissenserwerb ist die Voraussehbarkeit bzw. Kalku- 
lierbarkeit von Ereignissen und Zusammenhängen.

Lernen ist also eine komplexe Fähigkeit, die selbst des Lernens bedarf. Dies zeigt sich insbesondere im Prozess der Anwendung von gelerntem Wissen.

E-Learning stellt gegenüber schrifttextbasierten Lernmethoden in-sofern eine Besonderheit, dar, als angeforderte Informationen über elektronische Datenträger bzw. über online Datenbanken relativ schnell zur Verfügung gestellt und multimedial präsentiert werden können. Dies ist aus der Sicht der Wahrnehmungs-psychologie und der Bildungsökonomie ein entscheidender Vorteil gegenüber dem Schrifttext.

\subsection{Lernen und Handeln im Kontext computerbasierter Kommunikation}

Wenn man heute über Kommunikation spricht, dann werden in der Regel die neuen technischen Möglichkeiten assoziiert. Dies ist jedoch lediglich die oberflächliche Erscheinung eines tiefgreifenden Wandlungsprozesses. Die eigentlichen Folgen der Computer-Kommunikation werden sich vor allem im kommunikativen Handeln und im sozialen Verhalten der Menschen zeigen.

Dieser tiefgreifende Prozess hat alle modernen Industrienationen sowie Europa erfasst und das Schlagwort von der „elektronischen Demokratie“ erfährt eine neue Blüte. Vor allem in den USA, wo die „Teledemokraten“ „dem gegenwärtigen repräsentativen politischen System und den Wirkungen der politischen Berichterstattung in den Massenmedien“" die Schuld an Apathie, Frustration und Entfremdung der Wählerschaft geben [4]. Die sich nun formierten „Cyberdemokraten“ glauben, dass in den „, virtuellen Gemeinschaften „das verloren gegangene 'soziale Kapital $\square$ wieder gebildet werden kann". Nach ihrer Auffassung gewinnt der Einzelne dadurch einen Zuwachs an politischer Macht und wirtschaftlichem Wohlstand. Deshalb fordern die „Cyberdemokraten“ „die Förderung der kommunikativen Fähigkeit und den freien Zugang zu Informationen (a.a.O.: 67). Hier wird einmal mehr der Zusammenhang von technischen Möglichkeiten einerseits und sozialem und kommunikativem Verhalten andererseits deutlich.

Vor allem die Möglichkeit der kommunikativen Interaktion über das Internet wird das soziale Verhalten der Menschen verändern (vgl. dazu unten). Das neue Medium ermöglicht feed-back- Reaktionen wie in der realen Kommunikation. Und das ohne Zeitverzögerung.
Meinungsbildungsprozesse sind deshalb anderen Gesetzmäßigkeiten als noch vor 20 Jahren unterworfen. Die direkte Beteiligung des Bürgers an Umfragen und die schnelle Ergebnispräsentation fördern die Entwicklung bestimmter Erwartungshaltungen beim Bürger, so z. B. die nach der effektiven und schnellen Umsetzung von Politikentscheidungen. Auch wird die Zahl der Bürger zunehmen, die das Internet für die „Beteiligung“ am nationalen oder europäischen Meinungsbildungsprozess nutzen werden (vgl. dazu unten). Und die Zeit ist nicht fern, in der an die Stelle der Briefwahl das „E-Vote“ treten wird.

Auch der Begriff der „Öffentlichkeit“ muss neu definiert werden [5].

Die Grenze zwischen privater und öffentlicher Kommunikation wird immer schwerer zu ziehen sein. Ebenso sind neue Möglichkeiten des Missbrauchs entstanden. Den „Kommunikationsraum Internet“ [5] nutzen auch Rechtsradikale und Gegner der Demokratie.

Das schon früher angemahnte „kritische Textverständnis" [6] gewinnt angesichts dieser Entwicklungen eine völlig neue Bedeutung.

Der „Kommunikationsraum Internet“ fordert nicht nur Kommunikationswissenschaftler heraus, sondern ebenso Soziologen, Psychologen und Pädagogen, eine Didaktik der Internetkommunikation zu entwickeln, die eine Didaktik des E- Learning einschließen sollte. Ebenso ist die Handlungstheorie mit computervermittelten Handlungstypen konfrontiert, die zunehmend an Bedeutung gewinnen. So z. B.:

- Handlungen zur Informationsübertragung und Informationserwerb,

- Handlungen zum Erwerb von Wissen (Lernhandlungen),

- Handlungen zum Erwerb von Kompetenzen (kommunikative Kompetenzen, soziale Kompetenzen usw.),

- Handlungen zur Befriedigung von materiellen und ideellen Bedürfnissen (z. B. Erwerb von Waren, Erwerb von Dienstleistungen über das Internet, Unterhaltung).

Was die soziale Kompetenz angeht, so kann schon jetzt beobachtet werden, dass die „virtuelle Gruppe“ ein neuartiges Gruppenbewusstsein erzeugt. Allerdings mit der Gefahr, dass wichtige Kompetenzen für die Zusammenarbeit in der realen Gruppe vernachlässigt werden.

Johann Gottfried Herder, ein großer Europäer, bezeichnete sein Jahrhundert als ein „redendes und schreibendes" (vgl. dazu [6]). Unser Jahrhundert und 
das nächste erst Recht ist bzw. wird eines der multimedialen Kommunikation. Niemals zuvor hatten Informationen und deren Austausch eine solchen Einfluss auf den Menschen wie schon heute. Und nur, wer die Datenflut beherrscht und nicht von ihr beherrscht wird, kann aktives Subjekt dieser Entwicklung bleiben. Wer sagt dem Menschen von heute und morgen, was für ihn von Bedeutung ist, für ihn persönlich also einen „Wert“ hat. Was soll er behalten? Was kann er vergessen? Worüber sollte er kommunizieren, also schreiben und reden? Fragen, auf die es noch zuwenig Antworten gibt.

\subsection{Die Informationsflut beherrschen lernen}

E- Learning stellt im Kern „computergelenktes“ Lernen dar. Dies bedeutet nicht, dass auf den Einsatz von Tutoren verzichtet werden kann. Allerdings wird sich der E- Learning- Tutor hinsichtlich seiner Aufgaben wesentlich vom traditionellen Profil eines Lehrers unterscheiden müssen. Im Mittelpunkt seiner Arbeit wird die pädagogisch-didaktische Führung des Informationsvermittlungsprozesses zwischen Lerner und Computer stehen. Gerade um die Effektivität von E- Learning Prozessen zu erhöhen, ist der Einsatz entsprechender Experten für Informationsvermittlungsprozesse erforderlich.

Ihre Aufgabe wird es sein, Informationen nach pragmatischen Gesichtspunkten zusammenzufassen, Wichtiges von Unwichtigem zu trennen, Redundanz zu erkennen. Elektronische Suchmaschinen sollen dabei nur bedingt behilflich sein.

Das alleinige Anbieten von Informationen, zu welchem Sachgebiet auch immer, wird in der Zukunft nicht mehr ausreichen. Führungskräfte in Wirtschaft und Politik werden auf entsprechendes „Informationsmanagement" nicht mehr verzichten können. Führungsentscheidungen müssen zunehmend auf der Basis von aufbereiteten Informationspräsentationen getroffen werden. Derartige Präsentationen enthalten eine bestimmte, individuell auf den Nutzer zugeschnittene Auswahl von Informationen. Firmen, die ihre Wettbewerbsfähigkeit sichern wollen, werden sich dem Firmenprofil angepasste Datenbanken beschaffen müssen. Ebenso notwendig ist entsprechend geschultes Personal (vgl. unten).

Informationen und ihre Aufbereitung werden bereits jetzt als ,informationeller Mehrwert" gehandelt und werden so zu einem marktfähigen Produkt. Eine neue Chance für Existenzgründer und Fachkräfte tut sich auf [7]. Die Gründung eines europäischen Binnenmarktes für Informationsdienste signalisiert das
Entstehen eines neuen Industriezweiges in Europa. Welche Rolle Informationsverwertung und Kommunikation in der Zukunft für die wirtschaftliche Entwicklung von Regionen haben werden, ist schon heute erkennbar. Zurecht wird deshalb gefordert, unterentwickelte Regionen verstärkt in den internationalen Datentransfer einzubinden und verstärkt entsprechende Fachleute auszubilden. Solche Experten müssen mindestens folgende Aufgaben erfüllen:

- Erschließen von Informationen (vor allem unter Nutzung nationaler und internationaler elektronischer Datenbanken),

- Auswahl und Bewertung von Informationen,

- Präsentation, also Kommunikation von Informationen,

- Aufbau und Pflege von praktikablen und auf den Unternehmenszweck ausgerichteten Datenbanken.

Der „Infobroker“ (vgl. dazu [8]) wird also ausgeprägte analytische mit entsprechenden kommunikativen Fähigkeiten vereinen müssen.

Wie die Praxisbeispiele belegen, ist mit dem Infobroker ein Berufsbild entstanden, dessen Dienstleistung gerade für den sich rasch entwickelnden ELearning Sektor Perspektive haben wird. 1995 waren schon 260.000 Menschen im Bereich der Informationstechnologie beschäftigt, bis 2010 rechnet man mit weiteren 80.000 Arbeitsplätzen (vgl. a.a.O.: 29).

Mit derartigem Humankapital erhalten vor allem industriell schwach entwickelte Regionen eine zusätzliche Chance. Die EU fördert diese Entwicklung seit 1989 über das Programm IMPACT (vgl. dazu [9]).

\subsection{E- Learning als hypertextbasierter Wissenserwerb}

Der computererzeugte "Hypertext" stellt eine völlig neue Qualität der Informationspräsentation dar (vgl. dazu [10]). Hyperlinks stellen Verbindungen zu anderen Informationen her und steuern den Rezeptionsprozess. Diese „Links“ sind Mittel der Textnavigation. Nach Cicconi zeichnet sich der Hypertext durch folgende Merkmale aus:

1. Nodes ,that is a portion of the (hyper)text”.

2. Links "the lines connecting different portions of the hypertextual document" [11].

Ein wesentliches neues Moment der HypertextRezeption besteht darin, dass der Rezipient (,user") gleichzeitig Informationen verschiedener „Zeichenarten" aufnehmen muss, z. B. akustische, visuelle, farbliche Zeichen usw. Dies prägt ein Rezeptions- 
verhalten, dass auf Ganzheitlichkeit ausgerichtet ist (vgl. zur [12]).

Die Fähigkeit, geschriebene (gedruckte) Texte linear von links nach rechts zu rezipieren, wird nun durch die Fähigkeit der ganzheitlichen Hypertext-Rezeption ergänzt. Der traditionelle Text, die traditionelle Buchkultur, geht sicher nicht dem Ende, aber neuen Zeiten entgegen. Jochum und Wagner haben die wesentlichen Unterschiede zwischen geschriebenem Text und dem Hypertext zusammengefasst [13]: „Das Typographeum lässt sich durch eine offene Reihe von Merkmalen charakterisieren, die es vom EDV-Zeitalter abheben:

1. „Die feste Eingabe- und Ausgabestelle hat zur Folge, dass sich Rollen von Autor (auf der Eingabeseite) und Käufer bzw. Leser (auf der Ausgabeseite) ausdifferenzieren: Der Raum, der sich zwischen ihnen öffnet, schafft ein Informations- und Organisationsproblem, das der Buchhandel zu seiner Entwicklung genutzt hat.

2. Das Manuskript, das zur Transformation in den Druck gegeben wird, verweist zurück auf den Autor als geistigen Urheber oder Schöpfer.

3. Der Text in seinem ausgedruckten Zustand ist endgültig, unwandelbar und auf ewig fixiert. Die Metapher des Textes als Monument, im Handschriftenzeitalter geprägt, wird erst im Druck, der die Sachdimension des Textes hervorhebt - es ist immer dieser eine und kein anderer - voll eingelöst. Daher ist der Druckkultur das Muster von Primär- und Sekundärtext eigen (a.a. O.)“.

Diese Wesenszüge des schriftlichen Textes haben das kommunikative Handeln der Menschen über Jahrtausende geprägt und Wirtschaftszweige wie das Druckereigewerbe und das Verlagswesen begründet. Das Goethe-Wort: „Was man schwarz auf weiß besitzt , kann man getrost nach Hause tragen" bezeugt die fundamentale Bedeutung der "schwarzen Kunst" bis ins 18. Jahrhundert hinein. Die moderne Datenverarbeitung mit ihrer schwer nachvollziehbaren Dynamik produziert computergestützt Texte in hohen „Stückzahlen“. Der Computer kennt die Trennung von Eingabe- und Ausgabestelle nicht mehr. Oben genannte Autoren verwenden in diesem Zusammenhang die Bezeichnung „Fließtext“ (a.a.O.: 64).

Nach Kuhlen [7] hat der „Hypertext“ als Basis für den Wissenserwerb im Rahmen eines E-Learning-Konzeptes folgende Vorzüge:

- Flexibilität in der Darstellung,

- Prinzip der direkten Manipulation,

- direkter mehrdimensionaler Zu-griff,

- kognitive Plausibilität,
- individuelle Benutzeranpassung,

- leichte Änderbarkeit. (a.a.O.: 36-37).

E- Learning als moderne Form des Wissenserwerbs kann auch als computergestützte Produktion eines „,informationellen Mehrwertes“ beschrieben werden. Nach Kuhlen ist der ,informationelle Mehrwert in erster Linie Ergebnis methodisch kontrollierter Informationsarbeit" [7].

\section{Der User als neuer Lerntyp - Aspekte einer neuen Lernsoziologie}

Vor fast dreißig Jahren hat E. Klix in seinem Buch „Information und Verhalten“ umfassend die Rolle von Informationen bei der Verhaltenssteuerung dargestellt [1]. Weit vor ihm schrieb Morris sein berühmt gewordenes Buch „Signs, Language and Behavior“ [14]. Beide Autoren konnten damals nicht ahnen, welche tiefgreifenden Einflüsse die Computerära auf die Menschen des 20. Jahrhunderts haben würde. Unstrittig war aber schon für Morris und Klix der Zusammenhang von Information (Zeichen) und Handeln.

Informationsverarbeitung wird in diesem Sinn nach Klix als komplexer Prozess des Zusammenwirkens von externen und internen Faktoren aufgefasst. Die Informationsaufnahme erfolgt aus psychologischer Sicht auf der Grundlage eines oder mehrer Reize(s), der (die) entsprechende Prozesse auslöst bzw. auslösen.

Ein als Information erkannter Reiz wird erst in das Kurzzeitgedächtnis und dann nach einem internen Abgleichprozess in das Langzeitgedächtnis überführt, wenn das Subjekt dieser Information eine entsprechende Bedeutung beimisst (vgl. dazu [2]).

Die psychologischen Aspekte der textbasierten Informationsverarbeitung im Rahmen von Lernprozessen sind umfassend von Schnotz dargestellt worden, sie dürften prinzipiell auch auf den hypertextuell basierten Wissenserwerb zutreffen (vgl. [3]).

Es kann davon ausgegangen werden, dass der Wissenserwerb über hypertextuelle Präsentationen (ELearning) prinzipiell gleichen Gesetzen unterliegt wie der traditionelle Wissenserwerb über Schrifttexte. Gravierend ist jedoch der Einfluss von E -Learning Prozessen auf das Interaktionsverhalten des „users“. Unter der Überschrift ,weniger surfen, mehr lernen“ beschäftigt sich Alan Posener mit einem Essay von Todd Oppenheimer (USA) zum Thema „Computerwahn“. Er setzte sich mit den Argumenten der Computerbefürworter auseinander und kommt zu 
interessanten Schlüssen. So stellte er u. a. fest, dass das in den USA eingesetzte Leseprogramm „Reader Rabbit" zu einem 50-prozentigen Rückgang der Kreativität der Schüler geführt habe. Und weiter heißt es: "Unter den Erziehungswissenschaftlern wächst die Sorge, dass Kinder, die vor einem zweidimensionalen Bildschirm aufwachsen, sensorisch und sozial depriviert werden“" [15].

Dieser Aufsatz verweist auf die Gefahren des „Kommunikationsraums Internet“ wenn dessen Nutzung (vor allem bei Kindern und Jugendlichen) ohne entsprechende Anleitung verläuft. Aber auch andere Einflüsse auf das Verhalten der „user „sind schon erkennbar.

So verliert sich am Bildschirm trotz geographischer Entfernungen von oft tausenden von Kilometern das Gefühl der Distanz. Wenn man vor zwanzig Jahren telefonierte, konnte man noch Ferne ,ahnen“ Dieses Gefühl wird durch Distanzlosigkeit ersetzt. Distanzlosigkeit prägt zunehmend Vorstellungswelt, Denken und Handeln des „users“. Das deutsche „Sie“ als Ausdruck eines traditionellen Distanzrituals wird unter ,usern“ in der Regel nicht verwendet. Der Adressat einer Botschaft wird mit „Du“ angesprochen. Das unterstützt eine virtuelles Gruppengefühl. Tabus der realen Kommunikation werden tabufrei wie ein Blick in die Liste der „newsgroups“ beweist. So werden z. B. intime Sachverhalte im Chatroom öffentlich gemacht. Es mehren sich Fälle, in denen vor allem Jugendliche Orientierungen für die reale Welt verlieren.

Neben der realen Welt steht heute die virtuelle, die der realen zum Verwechseln ähnlich ist. Die interpersonale Kommunikation erfolgt auf zwei Ebenen der computervermittelten virtuellen und der traditionellen realen. In diesem Spannungsfeld von Virtualität und Realität erfolgt das kommunikative Handeln der Menschen im 21. Jahrhundert. Soziale Interaktion ist Voraussetzung und Folge gesellschaftlichen Daseins.

Jeder Mensch muss interagieren, will er sich eine Existenz in der modernen Industriegesellschaft sichern. Kommunikative und soziale Interaktion sind damit Grundelemente des sozialen Lebens überhaupt. Kommunikation und Interaktion sind Wesenszüge eines Menschen, der keine andere Chance zur Selbstverwirklichung hat, als über diesen Weg. Dennoch eröffnet das Internet dem Menschen völlig neue Perspektiven. Er kann schon heute einen Teil seiner zur Selbsterhaltung notwendigen Interaktionen über das Internet und die entsprechenden Serviceangebote sichern. Der Plausch im „Tante-EmmaLaden“, das Gespräch über Tagesereignisse mit dem Bäcker oder dem Autoschlosser könnten bald gänzlich der Vergangenheit angehören.

Virtuelle Klassenzimmer sollen nach dem Willen von Internet-Experten (vgl. oben) auch den Bildungsprozess entpersonalisieren. Aber kann in einem solchen Bildungsprozess Problemlösungskompetenz, soziales Handeln und Kreativität vermittelt werden. Dies ist schon jetzt nachdrücklich zu bezweifeln. Eine ganze Reihe von Grundbedürfnissen sind heute ohne direkte personale Interaktion regulierbar.

Aber andere, auf höherer Ebene angesiedelte Bedürfnisse des zivilisierten Menschen, kommen ohne direkte kommunikative Interaktion nicht aus. Dazu zählen u.a.:

- Aufbau und Festigung sozialert Kontakte (Freundschaften; Partnerschaften usw.),

- Entwicklung und Festigung des eigenen sozialen Status,

- Erringung und Festigung demokratischer gesellschaftlicher Verhältnisse

- Einsatz für verfassungsrechtliche Grundwerte (Meinungsfreiheit, Frieden, Völkerverständigung, Humanismus, Freiheit, Demokratie usw.).

Der Internetnutzer (,user“) ist ein sehr aktives Wesen. Er verfügt über ausgefeilte operationelle Fähigkeiten, z. B. kann er das Keyboard mit relativ hoher Geschwindigkeit bedienen und sich per Mausclick ein Informations- oder Unterhaltungsprogramm zusammenstellen usw. Je schneller er dies tut, um so mehr Informationen kann er pro Zeiteinheit „empfangen“ oder "senden“ lassen. In Fachkreisen wird in diesem Zusammenhang von „Clickrate“ gesprochen. Dieses operationelle Handeln muss präzise und zielgenau sein (vgl. dazu [16]).

In der virtuellen Welt organisiert er seine Gruppenbeziehungen (vgl. oben). Hier trifft er Bekannte und Freunde. Die Kommunikation innerhalb dieser Gruppen ist in der Regel frei von Asymmetrien. Vielmehr verkehrt der ,user“ mit gleichberechtigten Partnern. Er nutzt alle Optionen, wie überhaupt die Internetkommunikation vom Wesen her optional ist. So werden beständig verschiedene Alternativen der „Verlinkung“ offeriert. Der Nutzer befindet sich somit kommunikativ beständig in Such und Auswahlprozessen. Die Optionen des Netzes erzwingen beständig Reaktionen, genauer, entsprechende metakommunikative „user"-Handlungen. Derartige Handlungen stellen im eigentlichen Sinne kommunikationsorganisierende Handlungen dar, wie sie auch in der mündlichen und schriftlichen Kommunikation vorkommen. Der hohe Entscheidungs- und damit Handlungsdruck prägt das Verhalten dieses Menschen schon heute. 
Flexibilität, Effektivität und Zielgenauigkeit dürften Verhaltenseigenschaften sein, die „überkulturelle“ Geltung erlangen werden. Dieser „Lerntyp“ erwartet von der Politik schnelle und wirksame Entscheidungen. Auch Politik und Führung werden von diesen Menschen als eine Form der Optionsvermittlung begriffen, wie sie das Internet bietet. Dies hat Konsequenzen für die Führungsprozesse. Subordination und autoritärer Führungsstil haben sich spätestens mit dem Internet völlig überlebt. Und dieser „on- lineLerner" ist auch geschult im Umgang mit kommunikativen Regeln, die speziell im Internet gelten. Dies wird deutlich, wenn man die „Netikette“ der Chatter betrachtet. Es offenbart sich ein Kommunikationsraum mit eigenen Regeln. Das „Chatten“ ist inzwischen zu einer verbreiteten Form der e- mail Kommunikation geworden, die dem natürlichen Gespräch relativ nahe kommt und sich durch ein hohes „Interaktionspotential" auszeichnet (vgl. dazu [17]).

Jedoch gibt es entscheidende Unterschiede zur (natürlichen) face-to-face-Kommunikation zu berücksichtigen:

1. Die Chatter kommunizieren computervermittelt, also nicht direkt.

2. Die Chatter können keine nonverbalen Elemente der natürlichen Kommunikation (Gestik, Mimik) nutzen. (Sie behelfen sich jedoch mit graphischen „Sonderzeichen“ wie z. B.:J für smiley, < für traurig oder II für wütend usw.).

3. Die Kommunikation erfolgt hinsichtlich der thematischen Gestaltung (,newsgroups“) und hinsichtlich der Kommunikationsorganisation nach bestimmten Regeln.

Unter der Adresse: http://www.music.ch./about/faq/ internet-starter/einführung.html. heißt es u.a.: „Vergessen Sie nie, dass Sie es mit Personen zu tun haben. Oft werden Sie mit Personen anderer Kulturen kommunizieren. Denken Sie daran.“ Oder: „Benutzen Sie keine e-mail-Adressen für Werbung (Spamming genannt). Das verstößt in den meisten Ländern gegen das Gesetz... Lassen Sie sich nicht auf gegenseitige Beschimpfungen und Beleidigungen ein. Sie bringen nichts und belasten nur das Netz." Bei fast allen mailing lists und news groups gibt es „backups“, die man herunterladen kann. So heißt es weiter: „Durchsuchen Sie zuerst diese Backups, bevor Sie die InternetGemeinschaft zum tausendsten Mal mit derselben Frage langweilen. „Halten Sie sich an rechtliche Sachen wie Copyright, Vertrags- und Exportbestimmungen (a.a.O.). Satirisch gibt ein anderer „user“ Hinweise zum Chatverhalten: „Was der andere schreibt, ist grundsätzlich Mist..." An anderer Stelle folgt ein Hinweis auf folgende Kommunikations- techniken: „Den anderen als Kid darstellen, im Lehrerton schreiben, wenn der andere teilweise Recht hat, schließe auf keinen Fall einen Kompromiß oder gib dich tolerant. Nur im Streitthema solltest du beinhart sein" (Quelle: Reimer A. mellin mellin (anext.com). Als weitere Homepages zum kommunikativen Verhalten im Netz werden u. a. angeboten: Nettiquette, The Net User Guidlines and Netiquette, Netiquette und Core Rules of Netiquette. Wie man sehen kann, gelten im Internet durchaus vernünftige Diskurs-Regeln.

Die „virtuelle Kommunikationskultur“ hängt ganz sicher auch vom Bildungsniveau der „user“ ab. Er (der user) ist nach statistischen Angaben (vgl. unten) im allgemeinen überdurchschnittlich gebildet und regelbewusst.

\section{Zusammenfassung}

1. Lernen im Sinne von pädagogisch gesteuertem Wissenserwerb wird zukünftig maßgeblich computervermittelt erfolgen. Informationen werden durch gezielte Integration in vorhandene Speichersysteme zu individuell und (möglichst) langfristig verfügbarem Wissen.

2. E- Learning Prozesse zeichnen sich gegenüber schrifttextbasierten Lernprozessen durch schnelleren und effektiveren Informationstransfer zwischen dem Medium (Computer, Internet) und dem Lerner aus. Darin liegt die Tatsache begründet, dass E-Learning Prozesse durch speziell geschultes Personal angeleitet werden müssen. (E- Learning-Tutor).

3. Das hypertextuell basierte Lernen erleichtert die Vermittlung komplizierter Zusammenhänge. Vom Rezipienten erfordert dies die Beherrschung zweier verschiedener Rezeptionsformen:

a) die traditionelle lineare Rezeption von Schrifttexten,

b) die holistische (ganzheitliche) Erfassung von Kombinationen verschiedener semiotischer Systeme, die oftmals komplementär zusammenwirken, das heißt gemeinsam einen Inhalt begründen.

4. Der E-Lerner nutzt den „Kommunikationsraum Internet", um sich an Meinungsbildungsprozessen zu beteiligen. Dies wird vor allem durch die Interaktivität dieses Mediums möglich. Es entsteht zudem ein neue Qualität von „Öffentlichkeit“.

5. Die „virtuelle“ Kommunikation kann die „reale“ interpersonale lediglich ergänzen, jedoch nicht ersetzen. Vor allem Kinder und Jugendliche benötigen 
bei der Nutzung des Internets als Teil von E- Learning Prozessen Anleitung durch entsprechend geschultes Personal.

Die online- Kommunikation ermöglicht eine kommunikative Interaktion über große geographische Distanzen. Sie schafft ein Gefühl der Nähe und fördert ein spezielles Gruppenbewusstsein und verdrängt traditionelle Kommunikationstabus.

\section{Literatur}

1. Klix, F. (1980): Information und Verhalten, VEB Deutscher Verlag der Wissenschaften, Berlin.

2. Zimbardo, P (1988): Psychologie, 5. Aufl., Springer Verlag.

3. Schnotz, W. (1994): Aufbau von Wissensstrukturen. Untersuchungen zur Kohärenzbildung beim Wissenserwerb mit Texten, Beltz, Psychologie-Verlag, Union, Weinheim.

4. Hagen, M. (1999): Amerikanische Konzepte elektronischer Demokratie. In: Elektronische Demokratie, Hrsg. v. Klaus Kamps, 63-81.

5. Marschall, S. (1999): Alte und neue Öffentlichkeiten. Strukturmerkmale politischer Öffentlichkeit im Internet. In: Elektronische Demokratie, Hrsg. K. Kamps, Westdeutscher Verlag, 109-126.

6. Westphal, W. (1991): Überlegungen zur Entwicklung eines kritischen Textverständnisses als Aufgabe der modernen Schule. In: Sprachwissenschaft und Sprachkultur, Peter Lang, 378-399.
7. Kuhlen, R. (1991): Zur Theorie informationeller Mehrwerte. In: Killenberg u. a., 26-38.

8. Krauß-Leichert, U./ Schmidt, R. (Hrsg.) (1997): Dokumentarische Kompetenz in der MultimediaBranche, Verlag für Berlin-Brandenburg, Potsdam.

9. Huber, W. (1991): Die EG-Politik im Bereich des Informationsmarktes. In: Killenberg u. a., 11-25.

10. Lipczuk, R./ Mecner, P./ Westphal, W. (1999): Ausgewählte Begriffe zur modernen Linguistik und zur sprachlichen Kommunikation, Verlag Albatros, Szczecin

11. Cicconi, S. (1999): Hypertextuality. In: Mediapolis, Aspects of Texts, Hypertexts and multimedial Communication, Sam Inkinen (ed.), de Gruyter, Berlin, New York, 23-54.

12.Eco, U. (1991): Einführung in die Semiotik, München

13. Jochum, U./ Wagner, G. (Hrsg.) (1998): Am Ende das Buch. Semiotische und soziale Aspekte des Internet. Konstanz.

14. Morris, Ch. (1955): Signs, Language and Behavior, New York.

15. Posener, Alan, Die Welt, 24. 6. 2000: 4).

16. Hinner, K. (1998): Gesellschaftliche Auswirkungen moderner Kommunikationstechnologie am Beispiel des Internet, Logos- Verl.

17. Jakobs, E.-M. (1998): Online Zeitungen: Potentiale und Prozesse. In: Forum Angewandte Linguistik, Bd. 34, Peter Lang Verlag, 91-108. 